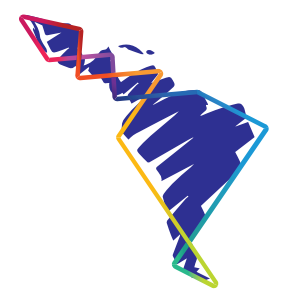

\title{
La importancia de la obligatoriedad para exigir el derecho a la educación en México
}

\author{
The importance of obligatoriness to \\ demand the right to education in \\ Mexico
}

\begin{abstract}
A importância da obrigatoriedade de exigir o direito à educação no México
\end{abstract}

\section{Mario Alberto Benavides-Lara}

\section{Resumen}

El presente texto ofrece un análisis conceptual acerca de las implicaciones que, en el marco de la legislación mexicana, tiene la obligatoriedad para impulsar a la educación como un derecho. El argumento central del artículo es que la obligatoriedad, más allá de un postulado normativo, es un mecanismo que permite la exigibilidad del derecho, que responde a un contexto sociopolítico determinado. Sin embargo, la forma en cómo el Estado mexicano ha atendido esta exigencia requiere un nuevo nivel de reflexión en el que las propuestas pedagógicas que surjan apuesten por un principio de reconocimiento a la diferencia que actualmente parece estar ausente.

Palabras clave: Acceso a la educación, derechos humanos, derecho a la educación, Estado y educación.

\section{Abstract}

The present text offers a conceptual analysis about the implications of the Mexican legislation framework as a tool of obligatoriness to advance education as a right. The central argument of the article is that obligatoriness, beyond being a normative postulate, is a mechanism that allows

1 Maestro en pedagogía. Coordinador de investigación en la Coordinación de Desarrollo Educativo e Innovación Curricular de la Universidad Nacional Autónoma de México. Correo electrónico: mario.benavides@ outlook.com. ORCID: https://orcid.org/0000-0003-4507-2078 
the enforceability of the right to education, which responds to a specific socio-political context. However, the way in which the Mexican state has met this demand requires a new level of reflection in which the pedagogical proposals that are to emerge, be committed to be committed with the recognition of difference and diversity that currently seems to be absent.

Keywords: Access to education; Human rights; Right to education; State and education.

\section{Resumo}

Este texto oferece uma análise conceitual sobre as implicações que, no marco da legislação mexicana, tem a obrigação de promover a educação como um direito. $\mathrm{O}$ argumento central do artigo é que a obrigação, para além de um postulado normativo, é um mecanismo que permite a exequibilidade de um direito, que responde a um contexto sociopolítico específico. No entanto, a forma em como o Estado mexicano atendeu a essa demanda exige um novo nível de reflexão em que as propostas pedagógicas que surgirem estejam comprometidas com um princípio de reconhecimento da diferença que atualmente parece estar ausente.

Palavras-chave: Acesso à educação; Direitos humanos; Direito à educação; Estado e educação.

\section{Introducción}

En este trabajo se presenta una exploración en torno a uno de los elementos estructurales del artículo tercero constitucional de la Constitución Política de los Estados Unidos Mexicanos (CPEUM), el cual establece el derecho a la educación en el país desde la constitución de 1917. Este elemento es la obligatoriedad de la educación, que detona la responsabilidad del Estado como garante de ésta en tanto derecho (DOF, 2018).

Para ello, en la primera parte del trabajo que corresponde a los apartados titulados "El estado mexicano frente a la reforma en derecho humanos", "Del reconocimiento al ejercicio del derecho" y "La obligatoriedad desde el derecho a la educación", se profundiza sobre el papel del Estado mexicano a partir de la reforma constitucional de 2011 en materia de derechos humanos y que incorpora el principio pro persona desde el que se reconoce la progresividad de los derechos, así como un nuevo marco de relación entre el Estado y la ciudadanía en el que adquiere sentido el derecho a la educación.

A fin de ofrecer al lector mayores elementos de comprensión de lo que implica este concepto de pro-persona, se hace un breve repaso de las características de los derechos humanos, centrándose especialmente en la característica de la progresividad, en tanto es ésta la que permite entender que el derecho a la educación es 
una construcción constante, y su obligatoriedad un mecanismo que permite dicha progresión, entendida como una expansión de derechos.

De esta manera, en la primera parte del apartado "Progresividad del derecho a la educación", se realizar un ejercicio de revisión que pone énfasis en la evolución e implicaciones que ha tenido, a partir de las reformas al artículo tercero, la expansión del principio constitucional de la obligatoriedad de la educación en las reformas de 1993 (obligatoriedad de la secundaria); 2002 (obligatoriedad del preescolar) y 2012 (obligatoriedad de la educación media superior). El objetivo de este recorrido será el dar cuenta de la forma en cómo ha cambiado la visión del Estado respecto de lo que se considera obligatorio en la educación desde una visión histórica, pero sobre todo conceptual.

Por último, en el apartado "La obligatoriedad en la educación. Nuevo desafíos pedagógicos", se plantea la necesidad de detonar una discusión en torno a nuevos referentes que, basados en el reconocimiento de la obligatoriedad, planteen una nueva lógica y los propósitos en torno al sentido pedagógico que adquiere la educación obligatoria (preescolar a media superior). Sobre este último punto se hacen dos acotaciones; el análisis que aquí se presenta no está articulado a una visión teórica única, es decir, no se hace un trabajo de análisis sociológico, jurídico o de ciencia política, sino que se trata de poner de relieve un concepto que, más que jurídico o histórico, pertenece al discurso de los derechos humanos.

En ese sentido, la contribución de este trabajo se encuentra en plantear el reto de lo que implica, a partir de entender las consecuencias de la obligatoriedad desde el enfoque de derechos humanos, la dimensión pedagógica de un sistema educativo que posee, en la obligatoriedad, un mecanismo de expansión de un servicio que es, primero que nada, un derecho. Sobre este punto se advierte que en este texto no se agota esta discusión, al contrario, sólo se presenta para su posterior desarrollo en otros documentos que el autor o quien esté interesado puede construir como parte de la discusión.

Metodológicamente, el texto se construyó a partir de una revisión documental para establecer la importancia del concepto de obligatoriedad en el discurso del derecho a la educación. Con base en esto se advierte al lector que, si bien no se ignora la complejidad de las condiciones de implementación que existen en el sistema educativo de México, la discusión que aquí se da tiene la intención de advertir la importancia de que una idea estructurante como es la obligatoriedad dé pie a que el sistema se mueva y organice, pasando con ello de lo conceptual a lo fáctico. 


\section{El Estado mexicano frente a la reforma en derechos humanos}

Actuar en clave de derechos humanos desde la realidad social, y no sólo desde la razón jurídica, implica reconocer la dignidad humana como condición intrínseca de la propia existencia (Habermas, 2010), pues es la base que iguala a las personas para gozar de una vida en sociedad que respete y reconozca la integridad de las personas y que permita, en consecuencia, el ejercicio de la llamada democracia de ciudadanía, es decir, la participación en la comunidad política en la que se da un proceso de expansión de derechos² (Beetham, 2007; OEA/PNUD, 2009).

La reforma a la Constitución mexicana en materia de derechos humanos que tuvo lugar en el año 2011 representa un avance en el reconocimiento de esta dignidad humana. Así como la democracia como un régimen político consustancial a la primera implica, por tanto, la transformación de la relación entre el Estado y la población a favor de esta última. Dicha redefinición de la relación entre el Estado y la ciudadanía está mediada por principios sociales, políticos, ideológicos, jurídicos, pero sobre todo éticos. Por tanto, como menciona Habermas (2010, p. 6), "los derechos humanos han sido producto de la resistencia al despotismo, la opresión y la humillación". La incorporación del principio pro-persona que está inicialmente plasmado en el artículo primero constitucional dimana y cohesiona el resto del articulado, en tanto es este el marco interpretativo desde el cual los derechos se hacen exigibles al Estado y se promueve su expansión (Medellín, 2013).

Artículo 1o. En los Estados Unidos Mexicanos todas las personas gozarán de los derechos humanos reconocidos en esta Constitución y en los tratados internacionales de los que el Estado Mexicano sea parte, así como de las garantías para su protección, cuyo ejercicio no podrá restringirse ni suspenderse, salvo en los casos y bajo las condiciones que esta Constitución establece. Párrafo reformado DOF 10-06-2011 Las normas relativas a los derechos humanos se interpretarán de conformidad con esta Constitución y con los tratados internacionales de la materia favoreciendo en todo tiempo a las personas la protección más amplia. Párrafo adicionado DOF 10-06-2011. (DOF, 2018, 1)

Este principio inclina la balanza de la justicia a favor de la ciudadanía y coloca los derechos humanos como la columna vertebral que debe guiar la acción del Estado. Tiene por resultado el reconocimiento de las autoridades de todos los niveles de gobierno y poderes como sujetos obligados y a la ciudadanía la coloca como sujetos de derechos (Carbonell, 2011).

2 En este texto se entiende por derechos a las libertades no sólo de tipo civiles sino del tipo de políticas, económicas, sociales y culturales que residen en el sujeto y en los pueblos. 
Estos cambios tienen implicaciones y retos connaturales a la adopción del discurso de los derechos humanos y, por tanto, a las características que definen su naturaleza como la indivisibilidad e interdependencia, la inalienabilidad e irrenunciabilidad, la universalidad, la progresividad, entre otras. A continuación, se ilustrará cómo se plasman estas características del derecho en aspectos concretos de éste, el cual será útil para comprender la importancia de que la educación sea reconocida desde la constitución como un derecho humano.

La primera característica que se destaca es la indivisibilidad en tanto todos los derechos son necesarios para alcanzar una vida digna e involucrar a las personas en la vida pública (Beetham, 1997). Esta indivisibilidad implica concebir al conjunto de los derechos humanos como interdependientes en tanto unos son condición de otros. Así, por citar un ejemplo, Beetham (1997) menciona que en el caso de los derechos económicos y sociales su negación tiene repercusiones en el ejercicio de los derechos civiles y políticos, lo cual se ve reflejado en la calidad de la democracia.

Siguiendo a este autor, si bien la economía o las políticas sociales no tienen una relación obvia con el régimen democrático en tanto el crecimiento económico, la prestación de servicios médicos, educativos o el empleo pueden ocurrir en regímenes políticos de corte autoritario. La diferencia con el ejercicio de derechos económicos, sociales y culturales, en una democracia, es que existe un entorno favorable para que se atiendan las necesidades de empleo, salud y educación no como concesiones que pueden ser suprimidas en cualquier momento por el poder político, sino como obligaciones que corresponden al Estado y que no pueden restringirse o suprimirse, ya que son inalienables e irrenunciables.

A estas características se suman la universalidad y la progresividad; la universalidad hace referencia a los derechos que se consideran fundamentales de manera independiente $^{3}$ a si son reconocidos o no dentro de un orden constitucional específico. Sin embargo, se mantienen como exigencias éticas que en el concierto internacional actúan como presión para que cada país los reconozca en su población; la reforma en derechos humanos mexicana es una muestra de ello (Vázquez y Serrano, 2011).

Por último, la progresividad, que es de especial interés para este texto, es la característica que de manera más contundente se relaciona con la idea de participación ciudadana y que se explorará en el siguiente apartado. La progresividad se entenderá como el mecanismo de expansión de derechos a partir del cual el Estado reconoce mayores libertades en la ciudadanía y que se derivan de la propia acción ciudadana

3 Respecto a la dificultad de definir qué es lo fundamental en tanto se parte que los derechos humanos son una construcción universal; Vázquez y Serrano (2011) hacen el llamamiento al diálogo intercultural de las civilizaciones para que sean ellas quienes definan esos derechos fundamentales. 
para que se dé este reconocimiento, el cual se refleja tanto en las políticas públicas como en las legislaciones correspondientes que incluyen el marco constitucional.

De acuerdo con Vázquez y Serrano (2011), la progresividad se ve reflejada en la implementación gradual de los derechos a partir de los planes (políticas públicas) encaminados a generar condiciones de implementación y mejoramiento en el disfrute de los derechos. En este sentido, la evolución del artículo tercero constitucional, en donde se reconoce el derecho a la educación, ha sido posible en gran medida a esta expansión o progresividad del derecho.

\section{Del reconocimiento al ejercicio del derecho}

Como ya se ha dejado constancia, la integración al marco constitucional de los derechos humanos en México ha representado, en los hechos, un cambio de paradigma que impacta a toda la organización social.

La centralidad que tiene para el Estado y el régimen político que la ciudadanía ejerza sus derechos se relaciona con que es a través de su exigibilidad que el modelo democrático se perfecciona y orienta a la equidad y genera condiciones de bienestar para la población y legitimidad para la democracia. Así, los derechos humanos se vuelven poderosas herramientas que cuando son invocados pueden ejercer presión para que los gobiernos respondan en el sentido demandado por la ciudadanía (Sen, 2000), lo cual se traduce en la construcción de un Estado más soberano en la medida en que atiende las necesidades de los sujetos gobernados.

De ahí la importancia de que los derechos se traduzcan en libertades en sentido positivo y no sólo en una reivindicación declarativa. Recuperando la idea de Amartya Sen, la libertad y los derechos, habrán de traducirse en libertades de poder; es decir, en las llamadas capacidades ${ }^{4}$, las cuales se definen aquí desde la lectura que a este planteamiento de Sen hace Rafael Cejudo (2007, p. 10) y que se entienden como "las oportunidades [que tiene la persona] para elegir y llevar una u otra clase de vida".

Para cerrar este apartado, es relevante mencionar que la utilidad que representa, en términos analíticos, el identificar esta articulación estriba en que se vuelve una herramienta adecuada para, como se verá en el siguiente apartado, ponderar la forma en cómo la legislación responde a estas necesidades de la población; específicamente en lo que hace al derecho a la educación en términos no sólo del derecho positivo sino de su realización.

$4 \quad$ En el discurso de Sen se utiliza el concepto de capabilities que en algunos casos se ha traducido como el neologismo capabilidades, para el caso de este texto se prefiere utilizar el término de capacidades. 


\section{La obligatoriedad desde el derecho a la educación}

En el artículo tercero constitucional se establece que "La educación preescolar, primaria y secundaria conforman la educación básica; ésta y la media superior serán obligatorias" (DOF, 2018). Esta declaración implica, en términos del derecho a la educación y apoyándose en el marco expuesto en el apartado anterior, que la educación, al ser obligatoria, además de ser reconocida como un derecho, es una responsabilidad que le corresponde al Estado y que, a diferencia de otros elementos o declaraciones contenidas en este mismo artículo, representa la característica en la que es más evidente el derecho a la educación, junto con la gratuidad y el grado de cumplimiento. Lo anterior, en la medida en que esta obligatoriedad se transforma en política pública y, en esa misma proporción, la exigibilidad de este rasgo de la educación es tangible.

La obligatoriedad como rasgo distintivo de la educación en México tiene un carácter histórico que da cuenta de la evolución política y social en el país. A pesar de que esta característica no se desprende del origen del artículo tercero, sí está presente como parte del articulado de la Constitución de 1917, específicamente en el artículo 31 (DOF, 1917, p. 25) que mencionaba en su redacción original:

Art. 31.- Son obligaciones de los mexicanos: I.- Hacer que sus hijos o pupilos, menores de quince años, concurran a las escuelas públicas o privadas, para obtener la educación primaria elemental y militar, durante el tiempo que marque la ley de Instrucción Pública en cada Estado.

Es de especial interés el observar cómo el carácter de la obligatoriedad de la educación se trasladó, de ser visto únicamente como una obligación por parte de las personas, a una obligación cuyo principal ejecutor y responsable es el Estado.

Siguiendo con el análisis sobre cómo la obligatoriedad se coloca en la conformación del sistema educativo mexicano, autores como Barba (2010) y Loyo (2010) mencionan que ésta se desprende del discurso de la modernización que surge, en el caso de México, como parte del proceso de construcción del Estado a partir del siglo XIX.

Aquí es importante detenerse a analizar el aparejamiento que existe entre educación y escolaridad en el contexto mexicano, aunque usualmente en la bibliografía pedagógica se parte de una definición convencional que distingue entre la educación formal, la no formal y la informal, y que hace referencia a los espacios en los que las personas reciben educación. Siguiendo a Loyo (2010), la obligatoriedad de la escuela supuso, para el caso de México, un cambio en la visión que desde el 
porfiriato tuvo el carácter optativo de la educación escolar que se contemplaba en la Ley de Instrucción Rudimentaria de 1911 a, una vez concluida la revolución, reconocer el carácter obligatorio que en principio se consagró exclusivamente en el artículo 31 de la Constitución de 1917; para, posteriormente, presentarse también como parte del artículo tercero de la educación elemental oficial y con ello sentar las bases de la conformación del Sistema Educativo Nacional (SEN).

A partir de estos antecedentes, se puede afirmar que la obligatoriedad no surge de un espíritu de progreso espontáneo o externo a la conformación sociopolítica e histórica de México. Sino que es una declaración en consonancia con el proyecto de país que se derivó de la oposición política y del movimiento social que dio origen a la revolución mexicana y que se consolidó en la Constitución del 17.

Cabe señalar que esta primera estructura que resultó de la reconfiguración política del país y que supuso, en su tiempo, una legislación de avanzada, ha ido transformándose y actualizándose a las demandas de los tiempos, agregando nuevas características hasta el punto de que, actualmente, el artículo tercero conforma el núcleo de un derecho a la educación que rompe con la mera idea de instrucción fundamental con el que había nacido y que en el texto original de 1917 apuntaba:

Art. 3o.-La enseñanza es libre; pero será laica la que se dé en los establecimientos oficiales de educación, lo mismo que la enseñanza primaria, elemental y superior que se imparta en los establecimientos particulares. Ninguna corporación religiosa, ni ministro de algún culto, podrán establecer o dirigir escuelas de instrucción primaria. Las escuelas primarias particulares sólo podrán establecerse sujetándose a la vigilancia oficial. En los establecimientos oficiales se impartirá gratuitamente la enseñanza primaria. (DOF, 1917, p. 50)

En este contexto en el que el derecho a la educación ha ido adquiriendo nuevos atributos, resulta de la mayor trascendencia que la educación, que regula el Estado, cumpla por sí misma con ciertas características de calidad y que, a la vez, responda directamente a este derecho a la educación obligatoria.

Sin embargo, parece que el más grande reto que implica el hacer efectiva la obligatoriedad de la educación se halla en la posibilidad real de la misma educación obligatoria de hacer sentido a los estudiantes y sus familias en el momento de tomar la decisión de que sus hijos e hijas acudan a las escuelas, y que se relaciona con la capacidad de la propia escuela y del sistema social de traducir la educación en oportunidades de movilidad social, o desde el pensamiento de Sen, que la educación permita desarrollar el bienestar de las personas. 
Parece, entonces, que la obligatoriedad de la educación consagrada en el artículo tercero se verá favorecida, apelando a la integralidad de los derechos humanos, en la medida en que otros derechos que están contemplados en el texto constitucional se materialicen en su ejercicio.

\section{Progresividad de la obligatoriedad en la educación}

El discurso del derecho a la educación, en el que se imbrica el asunto de la obligatoriedad, ha pasado, de acuerdo con Ruiz (2012), de la preocupación por lo legal a una discusión enfocada en lo educativo y a la forma en cómo este reconocimiento legal puede ser posible.

Esto ha llevado, de poner énfasis en la gratuidad y obligatoriedad -condiciones que se contempla forman parte del planteamiento legal o jurídico- a la preocupación por la exigibilidad y la justiciabilidad del derecho que hagan posible su ejercicio. Contrariamente, lo que en este texto se sostiene es que la obligatoriedad no se limita a una condición primaria del derecho a la educación que se resuelve en el texto normativo, sino que es el centro de este derecho, en tanto su definición exclusivista que distingue lo que sí es obligatorio y lo que no; permite valorar tanto su cumplimiento como desvelar las formaciones ideológicas que van orientando el discurso del Estado y de sus actores en relación con lo que es exigible en educación.

La expansión de la obligatoriedad no ha estado exenta de disputas, tensiones o análisis tanto de tipo político como académico; estas polémicas usualmente han estado dirigidas a la capacidad del Estado por atender este rasgo de la educación. Para dar cuenta de estas tensiones, a continuación se hará una breve revisión de cuáles han sido los principales obstáculos que, en su momento, se han identificado para incluir a cada uno de los niveles educativos en la definición de educación obligatoria 5 .

Cabe señalar que el sistema educativo mexicano se compone de cinco niveles, a saber: preescolar (3 años de duración), primaria (6 años de duración), secundaria (3 años de duración), educación media superior (3 años de duración); esto cuatro primeros niveles constituyen la educación obligatoria; el quinto nivel corresponde a la educación superior. Es importante señalar que la conformación de la educación obligatoria se ha dado de manera paulatina y a partir de la incorporación gradual de cada nivel, como se verá a continuación.

5 En este texto se omite la discusión en torno a la incorporación de la primaria, pues su reconocimiento como educación obligatoria forma parte de la conformación primera de la educación en el texto original del 17 y desde la cual se desplegaron los primeros esfuerzos por llevar escolarización al grueso de la población desde la naciente Secretaría de Educación Pública. 
En el caso de la obligatoriedad del preescolar, que tuvo lugar en el 2002, los principales argumentos que se esgrimían para cuestionarla se relacionaban con el incremento en términos de número de estudiantes para el sistema educativo y la incapacidad del Estado por resolver este asunto. Mencionaban Muñoz y Silva (2006) que esto podría afectar la calidad educativa, además de no quedar claro cuáles eran los beneficios que traería el inicio temprano de la escolaridad en la infancia.

Para el caso particular de secundaria, el proceso de reforma iniciado en el año de 1993, en el que se volvió obligatoria, implicó una actualización del modelo educativo. Mientras que durante muchos años lo usual era que la educación primaria fuera suficiente para asegurar una base común de conocimientos en la población, en la medida que la realidad laboral y social fue cambiando, la educación secundaria fue expandiéndose y proponiendo nuevos retos que implicaron una serie de reformas cuya intención fue actualizar el tipo de formación que se ofrecía en este nivel (Sandoval, 2000).

La obligatoriedad de la educación secundaria implicó, por tanto, el reconocer este nivel como un espacio académico y curricular cuya finalidad debe estar diferenciada de los dos niveles antecedentes (preescolares y primarios); así como del nivel que le sucede (media superior) y que en conjunto componen el tramo de la educación obligatoria. Actualmente, solventar esta diferencia, discusión que se mantiene abierta y vigente, permitirá dar cuenta de la necesidad pedagógica de este nivel, el cual ha estado fuertemente discutido (Ynclán, 1998).

Incorporados el nivel preescolar y secundaria a la escolarización obligatoria, el 9 de febrero de 2012 se publica en el Diario Oficial de la Federación (DOF) el decreto por el que se reforma el párrafo primero del artículo tercero constitucional. En este se añade, como parte del derecho a recibir educación, al nivel medio superior. Respecto a las críticas que han tenido lugar a partir de su incorporación como la parte final de la educación obligatoria, estas han estado principalmente orientadas a dos asuntos.

El primer tema de discusión respecto de la obligatoriedad de la educación media superior tiene que ver con la función que asume este nivel a partir de su nuevo estatus. Mientras que tradicionalmente era posible distinguir entre las opciones orientadas al trabajo y la oferta de educación media enfocada en la preparación propedéutica de estudiantes que ingresarían a la educación superior, con la obligatoriedad parte de la discusión ha estado dirigida a contrastar el sentido que se asume para este nivel y que estriba en la responsabilidad de que la formación que los estudiantes reciban les dote de los mínimos comunes en términos de aprendizaje y de los conocimientos que se espera que cualquier ciudadano mexicano posea (INEE, 2018b; Villa, 2010; Zorrilla, 2012). 
El otro punto que resalta de la obligatoriedad de la educación media es la que refiere nuevamente a la capacidad del Estado de poder atender la cobertura a partir de la demanda que se agregaría por cursar este nivel. Según lo relata Roberto Rodríguez (2012), la aprobación legislativa de esta nueva norma pasó por un periplo de seis años en los que los mayores argumentos y oposiciones de ciertos grupos de legisladores tuvieron que ver con la suficiencia presupuestal. Esta, después de diferentes negociaciones, se pudo destrabar estableciendo un proceso gradual de universalización del nivel vía artículos transitorios. Finalmente, la aprobación de esta reforma mereció, por parte del Presidente de la República, la siguiente declaración que recoge Rodríguez:

Con mucho orgullo, he firmado hoy, el Decreto por el cual se eleva a rango constitucional la Obligatoriedad de la Educación Media Superior. Se trata de un avance de la mayor trascendencia a la educación pública nacional. Con esta Reforma a nuestra Carta Magna, el Estado mexicano asume el compromiso de garantizar la enseñanza a todos nuestros niños y jóvenes, hasta el nivel de bachillerato. Esta medida, nos pone en el camino correcto para lograr, en el mediano plazo, un promedio nacional de escolaridad de 15 años de estudio, como la que tienen muchos de los países con los que competimos. (Rodríguez, 2012, p. 6)

A pesar de que la obligatoriedad de este nivel no es algo único en Latinoamérica, es importante ponderar que, independientemente de las limitaciones estructurales, como son la falta de cobertura física y geográfica en las ofertas existentes, así como la falta de docentes (INEE, 2019b), la obligatoriedad de la educación en México sienta nuevas bases que coloca retos analíticos similares a los que en su momento tuvo la educación secundaria, en tanto la EMS se vuelve el tramo último de la Educación Obligatoria.

Así se observa que muchos de los problemas, como son el aprovechamiento y el rezago en el que se encuentran los estudiantes de este nivel, que usualmente tenían gran visibilidad en el nivel secundaria, parecieran haberse transferido al nivel medio superior, acaso como consecuencia de que es la educación media la que da cuenta final del derecho a la educación obligatoria.

Recientemente, existe una propuesta legislativa para que la educación superior se vuelva obligatoria, no se ahonda en ella en tanto se desconocen los términos de esta obligatoriedad y en qué medida esto se está confundiendo con la idea de universalidad de la educación superior en la que México se encuentra rezagado en comparación con otros países en la región. 
Para cerrar este apartado, se ofrece información en la Tabla 1, relativa a la forma en cómo se asocian los datos de expansión de la cobertura de los cuatro niveles que conforman la educación obligatoria, a partir de los años en que se incorporaron con ese rasgo al párrafo primero del artículo tercero constitucional.

\section{Tabla 1. Expansión de los niveles educativos a partir de haberse decretado su obligatoriedad}

\begin{tabular}{|l|c|c|c|c|}
\hline \multicolumn{1}{|c|}{ Nivel } & Año en que & $\begin{array}{c}\text { Antes que se } \\
\text { decretara la } \\
\text { se decretó su } \\
\text { obligatoriedad }\end{array}$ & $\begin{array}{c}\text { Después } \\
\text { de que se } \\
\text { obligatoriedad } \\
\text { decretó la } \\
\text { obligatoriedad }\end{array}$ & \multirow{2}{*}{$\begin{array}{c}\text { Porcentaje } \\
\text { de expansión }\end{array}$} \\
\cline { 3 - 4 } & 2002 & $1^{\prime} 597,622^{6}$ & $2^{\prime} 939,840$ & 84.01 \\
\hline Preescolar & 1917 & $2^{\prime} 292,026$ & $3^{\prime} 564,767$ & 55.5 \\
\hline Primaria 7 & 1993 & $3^{\prime} 129,671$ & $3^{\prime} 924,610$ & 25.4 \\
\hline Secundaria & 2012 & $3^{\prime} 823,507$ & $4^{\prime} 682,336$ & 22.4 \\
\hline Educación Media Superior & & & &
\end{tabular}

Nota: Elaboración propia con base en datos de Serie histórica censal e intercensal 1990-2010 (INEGI, 2018) y Cifras Básicas INEE 2013 - 2014 (INEE, 2014).

Con base en los datos aquí presentados, y a pesar de que se puede argumentar que la expansión de la cobertura obedece a múltiples factores como la tendencia natural de crecimiento poblacional, lo que sí se puede hacer es establecer una hipótesis o supuesto en el que la obligatoriedad constitucional puede ser un factor permanente que empuja la cobertura de los servicios educativos. Contrario a los planteamientos de inicio, que como se vio remarcaban las deficiencias e incapacidades del Estado, el hecho de incorporarlo como normativa permite que las personas exijan su derecho a la educación. Queda por analizar, en otros estudios, cuál es la calidad de la oferta por medio de la cual el Estado ha cumplido con su obligación, así como valorar, especialmente en el caso de la educación media, si esta oferta es suficiente y pertinente (Guzmán, 2018).

\section{La obligatoriedad en la educación. Nuevos desafíos pedagógicos}

De acuerdo con el INEE, la educación, al volverse obligatoria, asume "la responsabilidad de incluir aquello que se considera fundamental en la formación de todo individuo" (INEE, 2016, p. 138). A partir de esta cita, es oportuno preguntarse

6 Estos datos corresponden al censo del año 2000, debido a que en este censo no se consideró la población escolar que asistía al preescolar, se toma la información de la población que contaba con 5 años y asistía a la escuela.

7 Para el caso de Primaria se retoman los datos del INEGI sobre los censos de 1911 y 1921, cabe aclarar que en ambos censos no existía una variable de escolarización sino de número de personas que sabían leer y escribir, mismos datos que se retoman para la conformación de esta Tabla. 
en qué consiste y cuáles son las consecuencias de establecer lo fundamental de la formación de las personas. Para ello, en este último apartado se abordará la obligatoriedad desde sus implicaciones pedagógicas, es decir, desde las consecuencias que tiene para el aprendizaje de las personas, el establecer un número mayor de años de escolaridad en la población.

Sobre este punto, si bien existe una crítica acerca del valor real de la escolarización para contribuir al bienestar de las personas, en matiz de esta idea, Gimeno (2000) menciona que aun y cuando la educación no es omnipotente para afrontar la desigualdad social, su no cumplimiento sí puede conducir a mayor desigualdad.

Lo anterior sirve de preámbulo para problematizar en uno de los temas que incluye la obligatoriedad y que se identifica como la homogeneización pedagógica implicada en la escolarización obligatoria. Mientras que de un lado está el asegurar una base común de aprendizajes para toda la población, atendiendo con esto la desigualdad; del otro lado se tiene que esta posición es conflictiva, en tanto supone una educación igual para todos los sujetos, lo cual lleva a ignorar las diferencias culturales, sociales y de visión, connaturales al ser humano (Infante y Matus, 2009). Estas situaciones, en el caso de México, ha sido muy habitual no considerarlas o tratarlas como indeseables.

Por poner un ejemplo, se tiene a Vasconcelos y su discurso pro-integración que afectó, desde una visión contemporánea de derechos humanos, los derechos culturales de los pueblos indígenas (Jiménez, 2011). Pero ipor qué este es un problema que se deriva de la obligatoriedad? Si se parte que todas y todos somos diferentes, la obligatoriedad que el Estado está mandatado a atender, y que pasa por asegurar la universalidad de los servicios educativos, se enfrenta, en la práctica, a la limitación de recursos que dificulta la atención de las desigualdades perniciosas y, en aras de ofrecer un servicio universal de educación, coloca, en cambio, en el centro de la estrategia del Estado, el optar por una educación que ignore estas diferencias no sólo culturales, sino socioeconómicas.

Frente a estos desafíos concretos que entrañan la obligatoriedad, ¿cuáles son las respuestas que desde los agentes educativos se les debe dar?, si se parte de que esta modificación en la Constitución ha significado un gran avance en el reconocimiento del derecho a la educación, queda por tratar de comprender las formas en que se traducen estos retos no sólo como políticas públicas sino desde la pedagogía.

La propuesta que hace Gimeno (2000) y que aquí se recupera como un insumo para pensar la obligatoriedad desde el trabajo de la escuela, parte de convocar a los diferentes agentes sociales para determinar, desde una consulta amplia y 
multilateral, a aquellos elementos que las escuelas deben cultivar a partir de definir las necesidades de aprendizajes de las personas que pasan por la educación obligatoria. En consecuencia, las funciones de la educación implican, según su nivel o especialidad, incorporar los contenidos que sean relevantes y comunes a la cultura, tanto para su manutención como para su trasformación (Benavides-Lara, 2015).

La escuela, entonces, deberá, en la lógica de brindar las bases de la formación de una ciudadanía común, fomentar los conocimientos que, debido a su naturaleza, no puedan ocupar otros espacios de la manera tan profunda y estructurada como se esperaría del establecimiento escolar (Gimeno, 2000).

De esta manera, la escuela asume un papel de agencia distribuidora de conocimientos que está llamada a atender las diferencias y, sobre todo, las desigualdades en tanto educación fundamental obligatoria; permite, con esto, el ejercicio de los derechos epistémicos que señala De Requena (2018). A la vez, provee a las personas de los contenidos fundamentales y comunes que les permitan incorporar en su subjetividad un conocimiento crítico-reflexivo del mundo, habilitándoles en la participación de su sociedad, pero con la potencialidad de transformarla. De ahí la importancia de que, en la construcción de respuestas, la educación obligatoria cumpla con objetivos que le den un sentido a la misma escolaridad obligatoria, que no conviertan, a la escolaridad, en una pirámide que tienda a la especialización, la verticalidad y, en esa misma lógica, a la exclusión social que sólo beneficia a quienes llegan a los más altos niveles de escolaridad (Gimeno, 2000).

En la Figura 1 se trata de ilustrar, de manera comparativa, la lógica piramidal actual de la educación obligatoria, comparada con un modelo ideal de lo que suponen los rasgos fundamentales y comunes de la obligatoriedad. Es de destacarse mientras que, en la lógica de pirámide invertida, o también podría decirse de embudo, lo que prima es una paulatina salida de estudiantes que no se ajustan a las características de cada nivel educativo o que bien, son expulsados por el sistema, lo cual se acrecienta en las poblaciones escolares con desventaja social. La lógica del cilindro supondría que, a pesar de estas diferencias, la escuela cumple el papel de igualadora o, al menos, conserva las diferencias procurando que todos reciban la educación fundamental y común. 
Figura 1. Las dos lógicas de la obligatoriedad

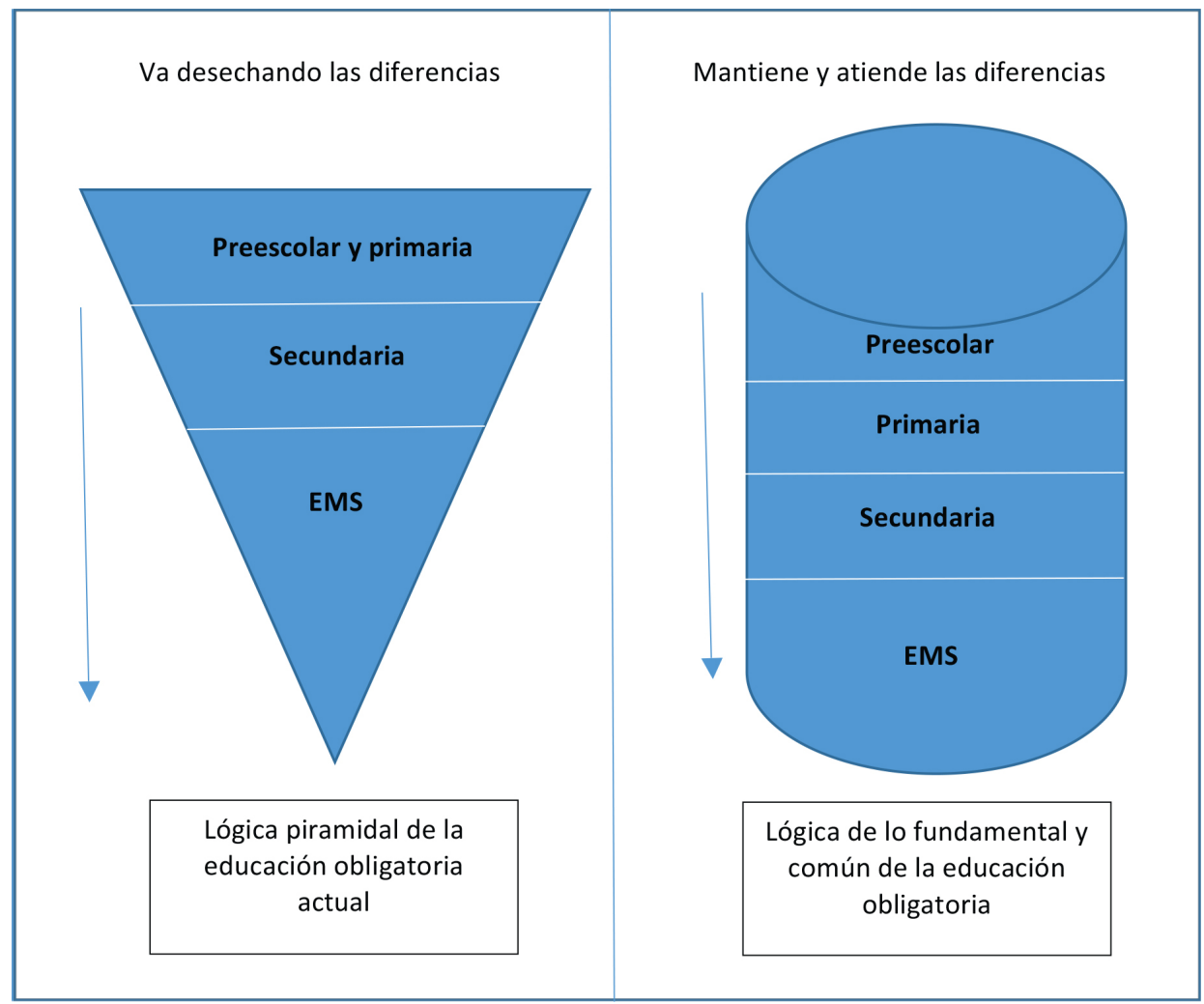

Nota: Elaboración propia.

\section{Conclusiones}

Como se puede ver, la expansión de la obligatoriedad ha seguido, cuando menos, dos lógicas. La primera se relaciona con el proceso de constitución del sistema educativo nacional a partir del México posrevolucionario, el cual se fijó como meta establecer un nuevo marco de reconocimiento de garantías sociales, el cual ha evolucionado hasta sentar las bases de una democracia de derechos que está siempre en construcción, pero que sirve como horizonte al cual todos los seres humanos aspiramos.

La otra lógica tiene que ver con la globalidad, la cual exige, por parte del sistema educativo, proveer de condiciones iguales de educación a su ciudadanía. Es así como la escolaridad en occidente se ha colocado como una meta que se busca alcanzar de manera independiente a una filiación ideológica. La globalidad, de alguna manera, ha hecho que esos conocimientos indispensables se expandan, 
diría Gimeno (2000), hacia una necesidad por penetrar y transformar la cultura en los aspectos que la escuela puede incidir.

Finalmente, desde una lectura transversal acerca de los argumentos que han dificultado la expansión de la obligatoriedad de la educación, se puede concluir que, si bien el Estado ha asumido su papel respecto del derecho a la educación, su actuación no ha estado exenta de temores y dudas sobre la efectividad del propio Estado por cumplir con esta obligación. A pesar de ello, la obligatoriedad ha sido un paso decisivo que se enfrenta a nuevos desafíos, los cuales requieren una mayor participación ciudadana, para, en conjunto, definir la educación fundamental, obligatoria y común que la ciudadanía necesita y merece.

\section{Referencias}

Barba, J. (2010). Los valores de la educación. En Arnaut, A. y Giourguli, S. (coord.), Los grandes problemas de México. Educación. COLMEX.

Beetham, D. (1997). Linking democracy and human rights. Peace Review, 9, 351 - 356. http:// doi/abs/10.1080/10402659708426076

Benavides-Lara, M. (2015). Flexibilizar el currículum de las instituciones de educación superior (IES) en un entorno de complejidad: Un análisis de casos desde la perspectiva cultural del socio-constructivismo. Voces y Silencios. Revista Latinoamericana de Educación 6, 1, 3 - 21. http:// DOI: 10.18175/vys6.1.2015.02

Carbonell, M. (2011). Las obligaciones del Estado en el artículo $1^{\text {ero }}$ de la Constitución mexicana. En Carbonell, M. y Salazar, P. (coord.), La reforma constitucional de derechos humanos: Un nuevo paradigma, 63-102, IIJ-UNAM.

Cejudo, R. (2007). Capacidades y libertad. Una aproximación a la teoría de Amartya Sen. Revista Internacional de Sociología, 47, 9-22.

De Requena Farré, J. A. (2018). Los derechos fundamentales epistémicos y comunicativos en la era de la posverdad. Revista Latinoamericana De Derechos Humanos, 29(2), 39-59. https:// doi.org/10.15359/rldh.29-2.2

DOF. (2018). Constitución Política de los Estados Unidos Mexicanos. http://www.diputados.gob. mx/LeyesBiblio/pdf/1_060320.pdf

DOF. (1917). Constitución Política de los Estados Unidos Mexicanos. https://archivos.juridicas.unam.mx/www/bjv/libros/6/2802/8.pdf

Gimeno, J. (2000). La educación obligatoria: Su sentido educativo y social. Ediciones Morata. 
Guzmán, G. C. (2018). Avances y dificultades en la implementación del Marco Curricular Común. Telebachillerato estatal, Educación Media Superior a Distancia y Telebachillerato comunitario. INEE.

Habermas, J. (mayo, 2010). El concepto de dignidad humana y la utopía realista de los derechos humanos. Diánoia, 64, 3 - 25.

INEGI. (2018). Serie histórica censal e intercensal 1990-2010 https://www.inegi.org.mx/ programas/ccpv/cpvsh

INEE. (2019). Condiciones básicas para la enseñanza y el aprendizaje en los planteles de educación media superior en México. Informe complementario. Autor.

INEE. (2018). Panorama Educativo de México 2017. INEE.

INEE. (2018b). La implementación del Marco Curricular Común en los planteles de la educación media superior. Autor.

INEE. (2016). La educación obligatoria en México. Informe 2016. INEE.

INEE (2014). Cifras básicas del Sistema Educativo 2013 y 2014. Autor. https://www.inee.edu. $\mathrm{mx} /$ evaluaciones/panorama-educativo-de-mexico-isen/mapa-2013-2014/

Infante, M., Matus, C. (2009). Policies and Practices on Diversity: Possibilities to Reimagine New Discourses. Disability and Society, 24(4), 437-445. https://doi. org/10.1080/09687590902879049

Jiménez, Y. (2011). Exclusión, asimilación, integración, pluralismo cultural y "modernización" en el sistema educativo mexicano: Un acercamiento histórico a las escuelas de educación pública para indígenas. Revista CPU-e, 12(1), 1-24. https://www.uv.mx/cpue/num12/ inves/completos/jimenez-exclusion-asimilacion.html

Loyo E. (2010). Fin del siglo y de un régimen. En Tanck, D. (coord.), La educación en México. COLMEX.

Medellín, X. (2013). Principio pro persona. Metodología para la enseñanza de la reforma constitucional en materia de derechos humanos. CDHDF / SCJN / ONU-DH. http://bibliohistorico. juridicas.unam.mx/libros/libro.htm?l=3033

Muñoz, C. y Silva, M. (2006). Análisis y resultados de las políticas públicas referidas a la educación básica. El caso de México. Trasatlántica de educación, 1, 99 - 117.

OEA/PNUD. (2009). La democracia de ciudadanía. Una agenda para la construcción de ciudadanía en América Latina. Autor. 
Ruiz, M. (2012). Derecho a la educación. Política y configuración discursiva. RMIE, 52, 39 - 64.

Rodríguez. R. (2012). La obligatoriedad de la educación media superior en México. Campus Milenio, 480, http://campusmilenio.mx/index.php?option=com_k2\&view=itemlist\&task=user\& id=866:robertorodr\%C3\%ADguezg\%C3\%B3mez\&Itemid=374

Sandoval, E. (2000). La trama de la escuela secundaria. PyV Editores.

Sen, A. (2000). El desarrollo como libertad. Gaceta ecológica, 55, 14-22.

Solís, P. (2015). Desigualdad social y efectos institucionales en las transiciones educativas. En Blanco, E. Solís, P. y Robles, H. (coord.), Caminos desiguales. Trayectorias educativas y laborales de los jóvenes de la Ciudad de México. INEE / COLMEX.

Vázquez, L. y Serrano, S. (2011). Los principios de universalidad, interdependencia, indivisibilidad y progresividad. Apuntes para su aplicación práctica. En Carbonell, M. y Salazar, P. (coord.), La reforma constitucional de derechos humanos: Un nuevo paradigma. IIJ-UNAM.

Villa, L. (2010). La educación media superior. En Arnaut, A. y Giourguli, S. (coord.), Los grandes problemas de México. Educación. COLMEX.

Ynclán, G. (1998). Todo por hacer. Algunos problemas de la Escuela Secundaria. Patronato SNTE para la Cultura del Maestro Mexicano A.C.

Zorrilla, J. (2012). Prioridades formativas para el bachillerato mexicano. Perfiles Educativos, $24,71-83$. 